\title{
Efficacy of deoxyspergualine for antibody- mediated rejection and proteinuria after kidney transplantation
}

\author{
Hideki Ishida \\ Kazunari Tanabe \\ Department of Urology, Tokyo \\ Women's Medical University, Tokyo, \\ Japan
}

\begin{abstract}
A patient showing antibody-mediated rejection (AMR) with severe vasculitis, and massive proteinuria after kidney transplantation was treated with deoxyspergualine (DSG, Spanidin $^{\circledR}$, Nippon Kayaku). DSG showed marked efficacy for this patient's symptoms. We report on a patient for whom DSG was effective against AMR after renal transplantation.

Keywords: deoxyspergualine (DSG), vascular rejection, antihuman leukocyte antigen (HLA) antibody
\end{abstract}

\section{Introduction}

Many aspects of the mechanisms of action of deoxyspergualine (DSG) remain unclear. Its remarkable efficacy has occasionally been reported not only in the field of transplantation but also in that of collagen diseases. DSG is considered to be an effective treatment for the morbid condition in which an immune response apparently causes proteinuria.

\section{Patient}

The patient was a 47-year-old male office worker.

\section{Past history}

Emergency hemodialysis as a result of pulmonary edema was initiated in 2001 to treat renal disease of unknown origin. The patient had not been followed up by physicians, although proteinuria was diagnosed 10 years previously. He was maintained on hemodialysis. There were no noteworthy complications other than hypertension.

\section{Clinical course (Figure I)}

The patient underwent kidney transplantation overseas on August 2003. His progress was checked in an outpatient ward in our department, starting in September 2003. The post-transplant immunosuppressive induction regimen consisted of cyclosporine (CsA), mycophenolate mofetil (MMF), and methylprednisolone (MP). Proteinuria was recognized as 500-800 $\mathrm{mg}$ total urine protein in a 24-hour collection one month after the transplant. Although a serum creatinine level $(\mathrm{sCr})$ of $1.1 \mathrm{mg} / \mathrm{dL}$ indicated a favorable function of the transplanted kidney, 6 months postoperatively on February 2004 , the urinary protein level tended to increase gradually, eventually reaching at least $3 \mathrm{~g}$ /day on April 2004. Steroid pulse therapy (500 $\mathrm{mg} \times 2$ ) was administered during this period, and at the same time CsA was temporarily changed to tacrolimus (FK506). 


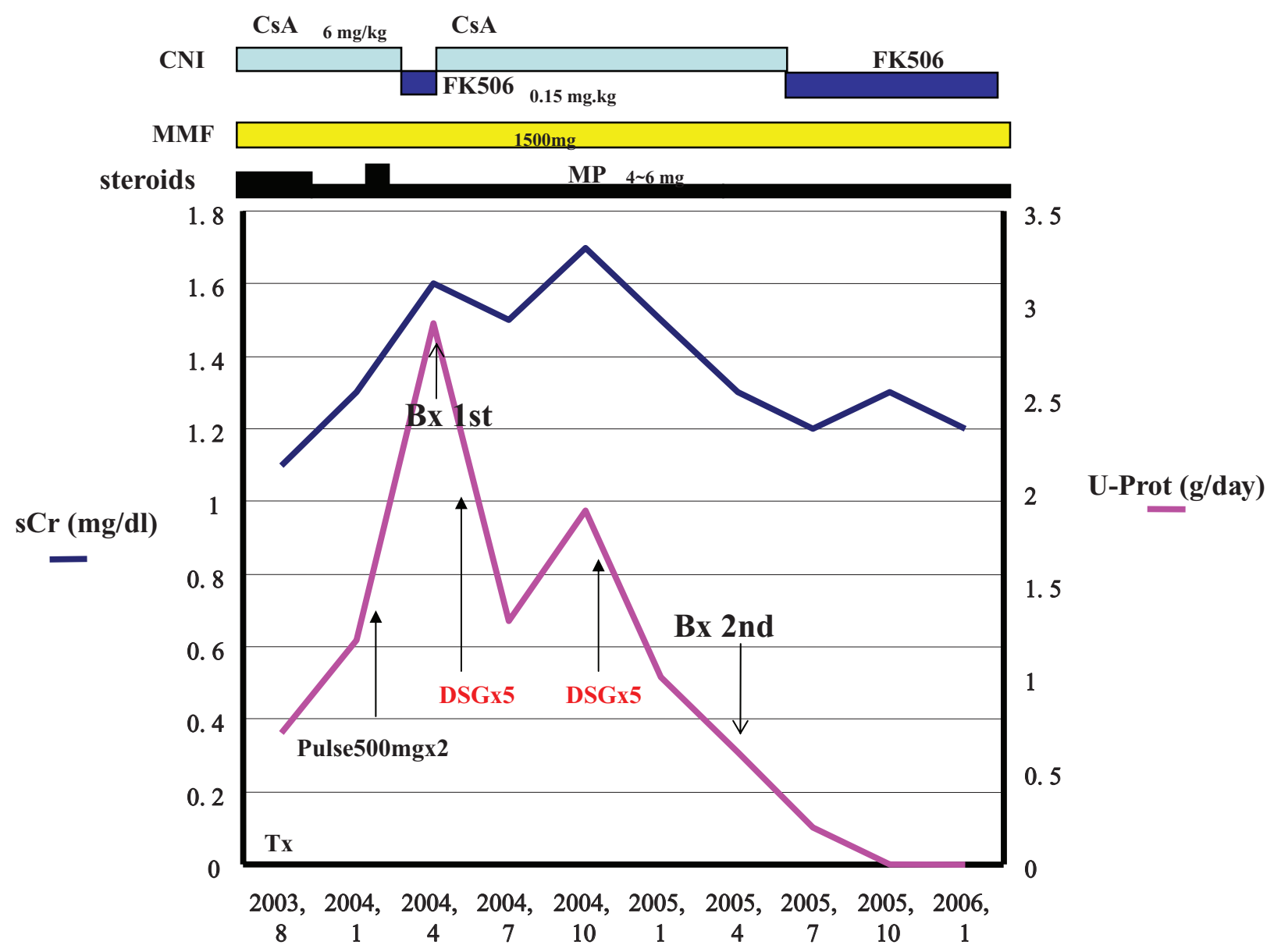

Figure I Clinical course. After the $2_{\text {nd }}$ dose of DSG treatment, a dramatic improvement in proteinuria was recognized, and urinary protein finally decreased to zero. On this occasion, renal biopsy was repeated. There was no evidence of a rejection and the vasculitis had improved markedly without $\mathrm{C} 4 \mathrm{~d}$ staining.

However, because the patient had severe neurological symptoms, FK506 was discontinued and CsA was restarted. From this time onward, sCr levels also rose gradually. Since $\mathrm{sCr}$ level increased from $1.2 \mathrm{mg} / \mathrm{dL}$ to $1.6 \mathrm{mg} / \mathrm{dL}$ on April 2004, the transplanted kidney was biopsied after the patient was hospitalized on May 2004. The biopsy revealed antibody-mediated rejection and severe vasculitis (Figure 2), and DSG was administered at a dose of $5 \mathrm{mg} / \mathrm{kg}$ for 5 days on June 2004. Thereafter, his condition was again checked in an outpatient ward. Because proteinuria increased with elevation of $\mathrm{sCr}$ from $1.4 \mathrm{mg} / \mathrm{dL}$ to $1.7 \mathrm{mg} / \mathrm{dL}$, despite a transient improvement, DSG was again administered at a dose of $5 \mathrm{mg} / \mathrm{kg}$ for 5 days on October 2004. After the second dose of DSG, a dramatic improvement in proteinuria was recognized, and urinary protein finally decreased to zero. On April 2005, renal biopsy was repeated. There was no evidence of a rejection and the vasculitis had improved markedly with disappearance of $\mathrm{C} 4 \mathrm{~d}$ deposition.
We switched CsA to FK506 on April 2005 again; however, the patient had no complaints such as neuralgia similar to the first switch. No antihypertensive drugs, such as angiotensin receptor blockers (ARB), were used during our patient's clinical course.

\section{Immunological examination}

Cross-matching test results before transplantation were all negative. With regard to post-transplant antibodies, Luminex (One Lamda, CA, USA) tests revealed the presence of massive amounts of A11, B41, and CREG was $1 \mathrm{C} 1$, but no changes in the types of antibodies before, compared with after, administration of DSG. We guess that $1 \mathrm{C} 1 \mathrm{CREG}$ are main donor-specific antibodies (DSA).

\section{Discussion}

Many aspects of the mechanisms of action of DSG remain unclear. However, its remarkable efficacy has occasionally 

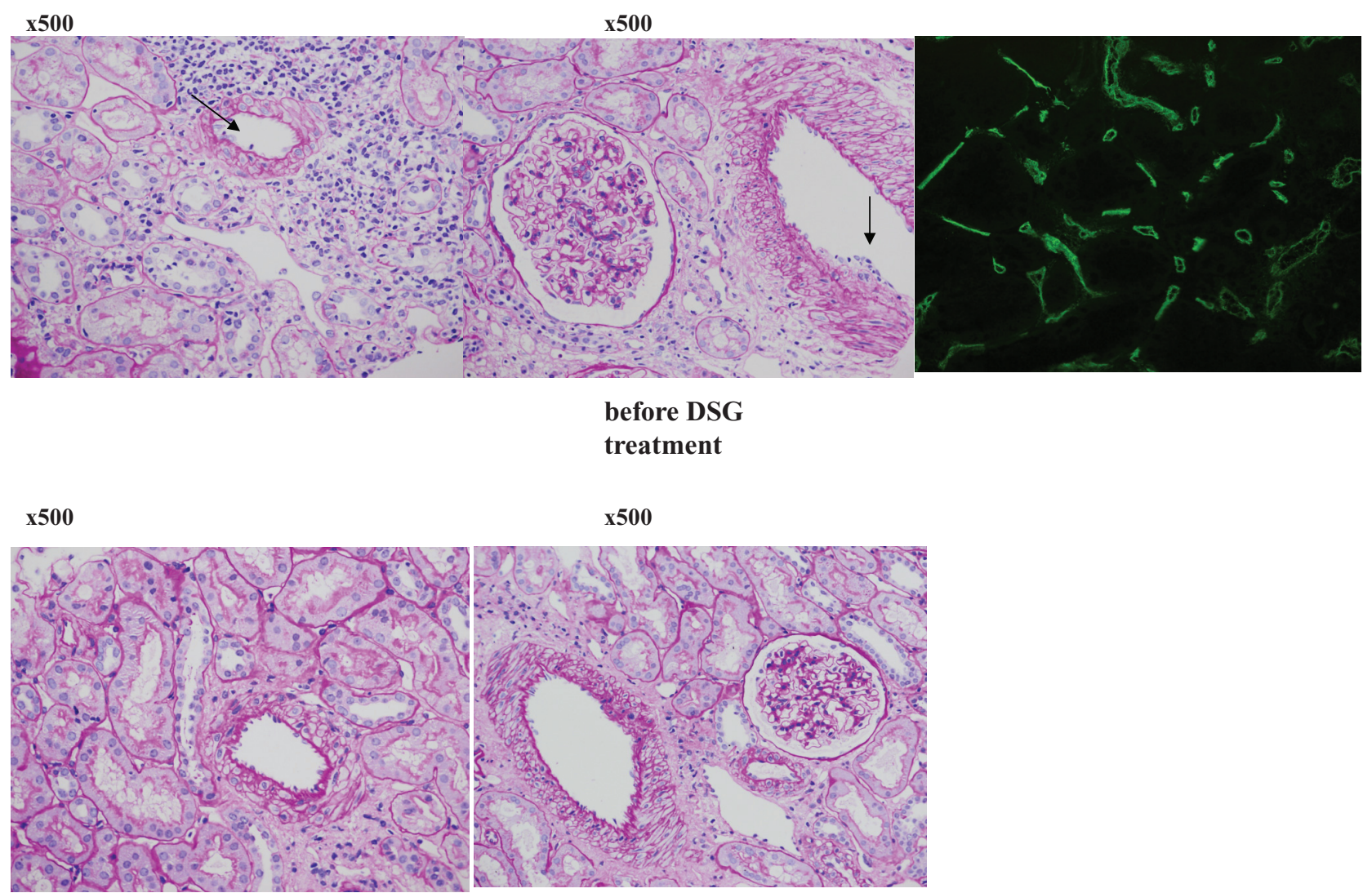

after DSG treatment

Figure 2 Pathological findings. Before DSG treatment:Specimen revealed aggressive cellular infiltration (black arrows) of arterial vessel wall and severe capillaritis and tubulitis, that suggests antibody mediated rejection with vasculitis. C4d deposition was also diffusely immunostained in peritubular capillaries. After DSG treatment: No rejection was shown in the specimen after DSG treatment. C4d deposition also disappeared after treatment.

been reported not only in the field of transplantation but also in that of collagen diseases. It seems that DSG exerts immunosuppressive effects that differ distinctly from those of CsA and FK506, which are calcineurin inhibitors (CNI), and from those of MMF, which are metabolic antagonists. Suppression of intracellular NF- $\mathrm{\kappa B}$ has been described as being representative of the mechanisms of DSG. In recent years, binding of DSG to an Hsp family (HSP 70, 90) molecule, a heat shock protein, was reported to suppress the antigen-presenting capacity of dendritic cells and the expressions of immunoglobulins on these cells (Nadler et al 1992, 1995).

There have been many reports on DSG-related improvements in clinical cases with severe vasculitis, which include Wegener granulomatosis and crescentic glomerulonephritis (Birck et al 2003; Shimitt et al 2005; Imai et al 2006). Researchers have also reported that all such patients showed severe vasculitis and cellular infiltration on biopsy, and remarkable improvements in these conditions after treatment with DSG. We consider that suppression of antibody-producing cells directly suppressed infiltrating cells in the kidney and also indirectly suppressed antibody production, both of which cause these conditions, thereby markedly improving the pathologic condition.

The advent of various immunosuppressive agents and the development of immunological monitoring have markedly improved outcomes of renal transplantation and have decreased post-transplant rejection, but we still encounter unexpected rejections. In particular, the rejection recognized in the present patient, ie, steroid-resistant severe antibody-mediated and vascular rejections, produced severe clinical symptoms, so that finding appropriate treatment was extremely challenging. Suppressing an immunologically activated condition, in which aggressive pathways involving interaction of $\mathrm{T}$ and $\mathrm{B}$ cells has already been triggered, leading to continuous production of anti-HLA antibodies, is very difficult. The usefulness of massive administration of $\gamma$-globulin and of conventional plasma exchange has also been more widely recognized, as has the efficacy of rituximab (anti-CD20 antibody). Saving the transplanted kidney 
by combining these methods has become more feasible. On the basis of our experience with the patient described herein, we consider that DSG should be considered as one of the treatment alternatives for such severe rejection reactions. The morbid condition, in which allogenic anti-HLA antibodies produced after transplantation directly attack the intravascular wall of the transplanted kidney, resembles the morbid condition of vasculitis in collagen diseases like Wegener's granulomatosis. DSG is also expected to be efficacious for such conditions.

\section{Conclusions}

We reported on a patient in whom DSG was effective against AMR after renal transplantation.

\section{References}

Birck R, Warnatz K, Lorenz HM, et al. 2003. 15-deoxyspergualine in patients with refractory ANCA-associated systemic vasculitis:a 6 months open label trial to evaluate safety and efficacy. J Am Soc Nephrol, 14:440-7.

Nadler SG, Eversole ACB, Tepper MA, et al. 1995. Elucidation the mechanism of action of the immunosuppressant 15-deoxyspergualine. Ther Drug Monit, 17:700-3.

Nadler SG, Tepper MA, Schacter B, et al. 1992. Interaction of the immunosuppressant deoxyspergualine with a member of the Hsp 70 family of heat shock proteins. Science, 258:484-6.

Shimitt WH, Birck R, Gobel U, et al. 2005. Prolonged treatment of refractory Wegener's granumatosis with 15-deoxyspergualine:an open study in seven patients. Nephrol Dial Transplant, 20:1083-92.

Imai H, Hotta O, Nose M, et al. 2006. Deoxyspergualine, an immunosuppressant, in patients suffering from nephropathies with crescent formation:an open label trial to evaluate safety and efficacy. Clin Exp Nephrol, 10:40-54. 